\title{
FINITE-DIMENSIONAL ODD HAMILTONIAN SUPERALGEBRAS OVER A FIELD OF PRIME CHARACTERISTIC
}

\author{
WENDE LIU ${ }^{\varpi}$ and YONGZHENG ZHANG
}

(Received 26 June 2003; revised 28 April 2004)

Communicated by J. Du

\begin{abstract}
Let $\mathscr{H}(m ; t)$ be the finite-dimensional odd Hamiltonian superalgebra over a field of prime characteristic. By determining ad-nilpotent elements in the even part, the natural filtration of $\mathscr{H}(m ; t)$ is proved to be invariant in the following sense: If $\varphi: \mathscr{H}(m ; \underline{t}) \rightarrow \mathscr{H}\left(m^{\prime} ; \underline{t}^{\prime}\right)$ is an isomorphism then $\varphi\left(\mathscr{H}(m ; t)_{i}\right)=\mathscr{H}\left(m^{\prime} ; \underline{t}^{\prime}\right)_{i}$ for all $i \geq-1$. Using the result, we complete the classification of odd Hamiltonian superalgebras. Finally, we determine the automorphism group of the restricted odd Hamiltonian superalgebra and give further properties.
\end{abstract}

2000 Mathematics subject classification: primary 17B50; secondary 17B40.

As is well known, filtration structures provide useful tools in the research of Lie algebras and Lie superalgebras. In particular, they play an important role in the classifications of finite-dimensional simple modular Lie algebras and finite-dimensional simple Lie superalgebras of characteristic zero respectively (see $[2,5,7,21,17]$ ). We know that Cartan-type Lie algebras and Lie superalgebras possess natural filtration structures. By means of invariance of filtrations one can characterize intrinsic properties of Cartan-type Lie algebras and Lie superalgebras and determine the automorphism groups (see $[22,16,24,26])$. In the case of Cartan-type modular Lie algebras, it is proved in [10] that the filtration of $X(m: \underline{1})$ is invariant under Aut $X(m: 1)$, where $X=W, S, H$ or $K$, and the same conclusion is obtained in [6] for all Cartan-type Lie algebras; by means of ad-nilpotent elements, the natural filtrations of infinite-dimensional Cartan-type Lie algebras are proved to be invariant under the automorphism groups (see [4]). In the case of characteristic zero, the natural filtrations of infinite-dimensional Lie algebras $X(m)$ is invariant, where $X=W, S, H$ or $K$ (see [14]). In [23] the author discussed the simplicity and restrictiveness of the

Supported by the National Natural Science Foundation of China.

(C) 2005 Australian Mathematical Society 1446-7887/05 $\$ A 2.00+0.00$ 
four classes of finite-dimensional modular Cartan-type Lie superalgebras. In [24] and [25], the invariance of natural filtrations of Hamiltonian superalgebras, generalized Witt superalgebras and special superalgebras are determined by means of image-space dimensions and ad-nilpotent elements, respectively.

In this paper, we discuss the finite-dimensional odd Hamiltonian superalgebra $\mathscr{H}(m ; t)$ over a field of positive characteristic. In the case of characteristic zero, the infinite-dimensional odd Hamiltonian superalgebra $\mathscr{H}(m, m)$, which is defined by odd Hamiltonian differential forms, is even transitive irreducible simple Lie superalgebra (see [8, Theorem 4.1]). This Lie superalgebra was interpreted as the Lie superalgebra of polyvector fields on an $m$-dimensional space (see [1]). It was introduced in [11] by Leites, and was later called Leites superalgebra (see [9]). Paper [12] gave a description of the outer derivations of this superalgebra.

We denote the natural filtration of $\mathscr{H}(m ; t)$ by $\{\mathscr{H}(m ; t), i \geq-1\}$. An isomorphism between any two odd Hamiltonian superalgebras is called $f$-isomorphism. In Section 2, we determine the ad-nilpotent elements with certain properties in the even part of $\mathscr{H}(m ; t)$. The results are used in Section 3 to prove that the filtration of $\mathscr{H}(m ; t)$ is invariant under any $f$-isomorphisms; that is, if $\varphi: \mathscr{H}(m ; t) \rightarrow \mathscr{H}\left(m^{\prime} ; t^{\prime}\right)$ is an isomorphism then $\varphi\left(\mathscr{H}(m ; t)_{i}\right)=\mathscr{H}\left(m^{\prime} ; \underline{t}^{\prime}\right)_{i}$ for all $i \geq-1$. As a result, we complete the classification of odd Hamiltonian superalgebras. In Section 4, we first prove the automorphism group of the restricted odd Hamiltonian superalgebra $\mathscr{H}$ is isomorphic to Aut $(\mathscr{U}: \mathscr{H})$, the admissible automorphism group of the base superalgebra $\mathscr{U}$. Then it is proved that the so-called standard normal series of Aut $\mathscr{H}$ is sent to the one of Aut( $\mathscr{U}: \mathscr{H}$ ). More detailed properties of Aut $\mathscr{H}$ are also discussed. The works in this section are motivated by the results and methods involved in Lie algebras (see $[19,20,4])$, and based on $[25$, Theorem 1].

\section{Preliminaries}

1.1. Notation and conventions The following notation and conventions are used throughout this paper:

- $\mathbb{F}$ denotes the underlying field of characteristic $p>2, \mathbb{Z}_{2}$ the ring of integers modulo 2; $\mathbb{N}$ and $\mathbb{N}_{0}$ the positive integer set and nonnegative integer set, respectively.

- Fix $m \in \mathbb{N} \backslash\{1,2\}$.

- $\mathrm{U}(m)$ denotes the divided power algebra over $\mathbb{F}$ with the $\mathbb{F}$-basis $\left\{x^{(\alpha)} \mid \alpha \in \mathbb{N}_{0}^{m}\right\}$.

- $\Lambda(m)$ denotes the Grassmann superalgebra in $m$ variables $x_{m+1}, x_{m+2}, \ldots, x_{2 m}$.

- Denote the tensor product by $\Lambda(m, m):=U(m) \otimes_{\mathrm{F}} \Lambda(m)$.

- We abbreviate $g \otimes f$ to $g f$ where $g \in \mathrm{U}(m), f \in \Lambda(m)$, and $x^{\left(\varepsilon_{i}\right)}$ to $x_{i}$, where $\varepsilon_{i}:=\left(\delta_{i 1}, \delta_{i 2}, \ldots, \delta_{i m}\right)$.

- Set $Y_{0}:=\{1,2, \ldots, m\}, Y_{1}:=\{m+1, m+2, \ldots, 2 m\}$ and $Y:=Y_{0} \cap Y_{1}$. 
- Set $B_{k}:=\left\{\left(i_{1}, \ldots, i_{k}\right) \mid m+1 \leq i_{1}<i_{2}<\cdots<i_{k} \leq 2 m\right\}, B(m):=\bigcup_{k=0}^{m} B_{k}$, where $B_{0}:=\emptyset$. For $u \in B_{k}$, put $|u|:=k,\{u\}:=\left\{i_{1}, \ldots, i_{k}\right\}, x^{u}:=x_{i_{1}} x_{i_{2}} \cdots x_{i_{k}}$, $x^{\natural}:=1$.

- Obviously, $\left\{x^{(\alpha)} x^{u} \mid \alpha \in \mathbb{N}_{0}^{m}, u \in B(m)\right\}$ is an $\mathbb{F}$-basis of $\Lambda(m, m)$.

- Define $D_{1}, \ldots, D_{2 m}$ to be linear transformations of $\Lambda(m, m)$ such that

$$
D_{i}\left(x^{(\alpha)} x^{u}\right)= \begin{cases}x^{\left(\alpha-\varepsilon_{i}\right)} x^{u} & i \in Y_{0} \\ x^{(\alpha)} \partial x^{u} / \partial x_{i} & i \in Y_{1},\end{cases}
$$

where $x^{\beta}:=0$ whenever $\beta \notin \mathbb{N}_{0}^{m}$.

- If $\operatorname{deg}(x)$ occurs in this paper, we always regard $x$ as a $\mathbb{Z}_{2}$-homogeneous element and $\operatorname{deg}(x)$ the $\mathbb{Z}_{2}$-degree of $x$.

- Define

$$
\mu(i):= \begin{cases}\overline{0} & i \in Y_{0} \\ \overline{1} & i \in Y_{1}\end{cases}
$$

- For $\underline{t}=\left(t_{1}, \ldots, t_{m}\right) \in \mathbb{N}^{m}$, put $\pi:=\left(\pi_{1}, \ldots, \pi_{m}\right)$ where $\pi_{i}:=p^{t_{i}}-1, i \in Y_{0}$, and $A(m ; t):=\left\{\alpha \in \mathbb{N}_{0}^{m} \mid \alpha_{i} \leq \pi_{i}, i \in Y_{0}\right\}$.

- Set

$$
i^{\prime}= \begin{cases}i+m & i \in Y_{0} \\ i-m & i \in Y_{1}\end{cases}
$$

- Let $\xi:=|\pi|+m=\sum_{i \in Y_{0}} p^{t_{i}}$.

1.2. The construction processes We know that $\Lambda(m, m)$ is an associative superalgebra with a $\mathbb{Z}_{2}$-gradation induced by the trivial $\mathbb{Z}_{2}$-gradation of $\mathrm{U}(m)$ and the natural $\mathbb{Z}_{2}$-gradation of $\Lambda(m)$. The following formulae hold in $\Lambda(m, m)$ :

$$
\begin{aligned}
x^{(\alpha)} x^{(\beta)} & =\left(\begin{array}{c}
\alpha+\beta \\
\alpha
\end{array}\right) x^{(\alpha+\beta)}, & & \alpha, \beta \in \mathbb{N}_{0}^{m} ; \\
x_{i} x_{j} & =-x_{j} x_{i}, & & i, j \in Y_{1} ; \\
x^{(\alpha)} x_{j} & =x_{j} x^{(\alpha)}, & & \alpha \in \mathbb{N}_{0}^{m}, j \in Y_{1} .
\end{aligned}
$$

Clearly, $D_{1}, \ldots, D_{2 m}$ are superderivations of $\Lambda(m, m)$. Let

$$
W(m, m)=\left\{\sum_{i \in Y} a_{i} D_{i} \mid a_{i} \in \Lambda(m, m), i \in Y\right\} .
$$

Then $W(m, m)$ is an infinite-dimensional Lie superalgebra (see [23]), which is a subalgebra of $\operatorname{Der}_{F}(\Lambda(m, m))$. We note that $W(m, m)$ is free $\Lambda(m, m)$-module with a $\Lambda(m, m)$-basis $\left\{D_{1}, \ldots, D_{2 m}\right\}$. 
The following formula holds in $W(m, m)$ :

(1) $[a D, b E]=a D(b) E-(-1)^{\operatorname{deg}(a D) \operatorname{deg}(b E)} b E(a) D+(-1)^{\operatorname{deg}(D) \operatorname{deg}(b)} a b[D, E]$.

Consequently,

$$
\left[a D_{i}, b D_{j}\right]=a D_{i}(b) D_{j}-(-1)^{\operatorname{deg}\left(a D_{i}\right) \operatorname{deg}\left(b D_{j}\right)} b D_{j}(a) D_{i}
$$

where $a, b \in \Lambda(m, m), D, E \in W(m, m), i, j \in Y$.

From the definition of $A(m ; \underline{t})$, we obtain that

$$
\Lambda(m, m ; \underline{t}):=\operatorname{span}_{F}\left\{x^{(\alpha)} x^{u} \mid \alpha \in A(m ; \underline{t}), u \in B(m)\right\}
$$

is a finite-dimensional subalgebra of $\Lambda(m, m)$. Set

$$
W(m, m ; t)=\left\{\sum_{i \in Y} a_{i} D_{i} \mid a_{i} \in \Lambda(m, m ; t), i \in Y\right\},
$$

then $W(m, m ; t)$ is a finite-dimensional subalgebra of $W(m, m)$ (see [23]).

Define $\mathrm{T}_{\mathrm{H}}(a)=\sum_{i \in Y}(-1)^{\mu(i) \operatorname{deg}(a)} D_{i}(a) D_{i^{\prime}}$, where $a \in \Lambda(m, m ; t)$. Then $\mathrm{T}_{\mathrm{H}}$ is an odd linear mapping from $\Lambda(m, m ; t)$ to $W(m, m ; t)$, that is, $\mathrm{T}_{\mathrm{H}}\left(\Lambda(m, m ; t)_{\theta}\right) \subset$ $W(m, m ; t)_{\theta+\overline{1}}$, for $\theta \in \mathbb{Z}_{2}$. Let $\mathscr{H}(m ; t)=\left\{\mathrm{T}_{\mathrm{H}}(a) \mid a \in \Lambda(m, m ; t)\right\}$. Then $\mathscr{H}(m ; t)$ is a subalgebra of $W(m, m ; t)$, which is called odd Hamiltonian superalgebra (see [8, page 27]). We have the following formula (see [8, page 28]):

$$
\left[\mathrm{T}_{\mathrm{H}}(a), \mathrm{T}_{\mathrm{H}}(b)\right]=\mathrm{T}_{\mathrm{H}}\left(\mathrm{T}_{\mathrm{H}}(a)(b)\right) .
$$

Recall the natural $\mathbb{Z}$-gradations of $\Lambda(m, m ; t)$ and $W(m, m ; t)$ :

$$
\begin{aligned}
\Lambda(m, m ; t) & =\bigoplus_{i=0}^{\xi} \Lambda(m, m ; \underline{t})_{[i]}, \quad \text { where } \\
\Lambda(m, m ; t)_{[i]} & =\operatorname{span}_{\mathbb{F}}\left\{x^{(\alpha)} x^{u}|| \alpha|+| u \mid=i, \alpha \in A(m ; \underline{t}), u \in B(m)\right\} \\
W(m, m ; t) & =\bigoplus_{i=-1}^{\xi-1} W(m, m ; \underline{t})_{[i]}, \quad \text { where } \\
W(m, m ; t)_{[i]} & =\operatorname{span}_{\mathbb{F}}\left\{a_{j} D_{j} \mid a_{j} \in \Lambda(m, m ; \underline{t})_{[i+1]}, j \in Y\right\} .
\end{aligned}
$$

It is easy to verify that $\mathscr{H}(m ; t)$ is a $\mathbb{Z}$-graded subalgebra of $W(m, m ; t)$

$$
\begin{aligned}
\mathscr{H}(m ; t) & =\bigoplus_{i=-1}^{\xi-2} \mathscr{H}(m ; t)_{[i]}, \quad \text { where } \\
\mathscr{H}(m ; t)_{[i]} & =\mathscr{H}(m ; t) \cap W(m, m ; t)_{[i]} \\
& =\left\{\mathrm{T}_{\mathbf{H}}(a) \mid a \in \Lambda(m, m ; t)_{[i+2]}\right\} .
\end{aligned}
$$


Set $W(m, m ; \underline{t})_{i}=\bigoplus_{j \geq i} W(m, m ; \underline{t})_{[j]}, \mathscr{H}(m ; \underline{t})_{i}=\bigoplus_{j \geq i} \mathscr{H}(m ; \underline{t})_{[j]}$. Recall that $\left\{W(m, m ; t)_{i}, i \geq-1\right\}$ and $\left\{\mathscr{H}(m ; \underline{t})_{i}, i \geq-1\right\}$ are said to be the natural filtrations of $W(m, m ; t)$ and $\mathscr{H}(m ; t)$, respectively.

From now on, we frequently abbreviate $W(m, m ; t)$ and $\mathscr{H}(m ; t)$ to $W$ and $\mathscr{H}$, respectively.

\section{The ad-nilpotent elements in $\mathscr{H}_{\overline{0}}$}

Let $L$ be a Lie superalgebra and $S$ a nonempty subset of $L$. Recall that an element $x$ of $S$ is called ad-nilpotent, if $\operatorname{ad} x$ is a nilpotent linear transformation of $L$. We denote by nil $(S)$ the set of ad-nilpotent elements in $S$.

For $\mathscr{H}(m ; t)$ where $m \in \mathbb{N} \backslash\{1,2\}$ and $\underline{t} \in \mathbb{N}^{m}$, define

$$
\begin{aligned}
& \Omega:=\left\{E \in \operatorname{nil}\left(\mathscr{H}_{\overline{0}}\right) \mid(\operatorname{ad} E)(\mathscr{H}) \subset \operatorname{nil}(\mathscr{H})\right\} \\
& \Gamma:=\left\{E \in \operatorname{nil}\left(\mathscr{H}_{\overline{0}}\right) \mid(\operatorname{ad} E)(\Omega) \subset \Omega\right\} \\
& \Phi:=\left\{E \in \mathscr{H} \mid(\operatorname{ad} E)\left(\mathscr{H}_{1} \cap \mathscr{H}_{\overline{0}}\right) \subset \operatorname{nil}(\mathscr{H})\right\} .
\end{aligned}
$$

Let $m^{\prime} \in \mathbb{N} \backslash\{1,2\}, \underline{t^{\prime}} \in \mathbb{N}^{m^{\prime}}$. For $\mathscr{H}\left(m^{\prime} ; \underline{t}^{\prime}\right)$, the corresponding sets are denoted by $\Omega^{\prime}, \Gamma^{\prime}$ and $\Phi^{\prime}$, respectively.

Proceeding analogously to [18, Theorem 1.3 .1$]$ or [3, Theorem 2.1], we may prove the following lemma.

LEMMA 2.1. Let $L$ be a finite-dimensional Lie superalgebra, and $S$ a Lie subset of $L$, that is, $S$ is closed under the multiplication of $L$. If $S \subset \operatorname{nil}(L)$, then $\operatorname{span}_{\mathbb{F}} S \subset \operatorname{nil}(L)$.

For $\mathbb{Z}$-graded Lie superalgebras we have the following lemma.

LEMMA 2.2. Let $L$ be a $\mathbb{Z}$-graded Lie superalgebra. Suppose that $x \in \operatorname{nil}(L)$. Then $\mathrm{m}_{\mathbb{Z}}(x) \in \operatorname{nil}(L)$, where $\mathrm{m}_{\mathbb{Z}}(x)$ is the nonzero $\mathbb{Z}$-component of $x$ possessing the minimal $\mathbb{Z}$-degree.

ProOF. See [25, Lemma 2].

Now we return to the case of $\mathscr{H}(m ; t)$.

Lemma 2.3. Suppose that $a \in \Lambda(m, m ; t)$. Then $\mathrm{T}_{\mathrm{H}}(a) \in \operatorname{nil}(\mathscr{H})$ if and only if $\mathrm{T}_{\mathrm{H}}(a)$ is a nilpotent transformation of $\Lambda(m, m ; t)$.

ProOF. Let $b \in \Lambda(m, m ; t)$. Applying (2) we obtain by induction on $k$ that

$$
\left(\operatorname{ad} \mathrm{T}_{\mathrm{H}}(a)\right)^{k}\left(\mathrm{~T}_{\mathrm{H}}(b)\right)=\mathrm{T}_{\mathrm{H}}\left(\left(\mathrm{T}_{\mathrm{H}}(a)\right)^{k}(b)\right) \text { for all } k \in \mathbb{N} \text {. }
$$

Combining this with the fact $\operatorname{Ker} T_{H}=\mathbb{F} \cdot 1$, we obtain the desired result. 
Since $\mathscr{H}$ is finite-dimensional, it is clear that $\mathscr{H}_{[-1]} \cup \mathscr{H}_{1} \subset \operatorname{nil}(\mathscr{H})$. For the ad-nilpotent elements of $\mathscr{H}_{[0]}$, we have the following result.

LEMMA 2.4. Let $i, j \in Y$. Then $\mathrm{T}_{\mathrm{H}}\left(x_{i} x_{j}\right) \in \operatorname{nil}(\mathscr{H})$ if and only if $i^{\prime} \neq j$.

ProOF. By the definition of $T_{H}$, we have

$$
\mathrm{T}_{\mathrm{H}}\left(x_{i} x_{j}\right)=(-1)^{\mu(i)+\mu(i) \mu(j)} x_{j} D_{i^{\prime}}+(-1)^{\mu(j)} x_{i} D_{j^{\prime}} .
$$

Clearly, $x_{i}^{p}=x_{j}^{p}=0$. Suppose that $i^{\prime} \neq j$. It is easy to see that $\left(x_{j} D_{i^{\prime}}\right)^{p}=$ $\left(x_{i} D_{j^{\prime}}\right)^{p}=0$. From $\left(1^{\prime}\right)$, we have $\left[x_{j} D_{i^{\prime}}, x_{i} D_{j^{\prime}}\right]=0$. In combination with (3), we have $\left(\mathrm{T}_{\mathrm{H}}\left(x_{i} x_{j}\right)\right)^{2 p}=0$. By virtue of Lemma 2.3 , we obtain that $\mathrm{T}_{\mathrm{H}}\left(x_{i} x_{j}\right) \in \operatorname{nil}(\mathscr{H})$, as desired.

Conversely, assume that $\mathrm{T}_{\mathrm{H}}\left(x_{i} x_{j}\right) \in \operatorname{nil}(\mathscr{H})$ with $i^{\prime}=j$. Without loss of generality, we may assume that $i \in Y_{0}$. By (3), $\mathrm{T}_{\mathrm{H}}\left(x_{i} x_{i^{\prime}}\right)=x_{i^{\prime}} D_{i^{\prime}}-x_{i} D_{i}$. Note that

$$
\left(\mathrm{T}_{\mathrm{H}}\left(x_{i} x_{j}\right)\right)^{k}\left(x_{i^{\prime}}\right)=x_{i^{\prime}} \quad \text { for all } k \in \mathbb{N} \text {. }
$$

Therefore, $\mathrm{T}_{\mathrm{H}}\left(x_{i} x_{i^{\prime}}\right)$ is not a nilpotent transformation of $\Lambda(m, m ; t)$, which contradicts Lemma 2.3.

LEMMA 2.5. Suppose that $E_{[0]} \in \operatorname{nil}\left(\mathscr{H}_{[0]}\right)$ and $\left[E_{[0]}, E_{[0]}\right]=0$. Then $E_{[0]}+E_{1} \in$ $\operatorname{nil}(\mathscr{H})$ for all $E_{1} \in \mathscr{H}_{1}$.

Proof. Clearly, $\left\{E_{[0]}\right\} \cup \mathscr{H}_{1}$ is a Lie subset of $\mathscr{H}$, in which all elements are adnilpotent. By Lemma 2.1, $\operatorname{span}_{\mathbb{F}}\left(\left\{E_{[0]}\right\} \cup \mathscr{H}_{1}\right) \subset \operatorname{nil}(\mathscr{H})$. In particular, $E_{[0]}+E_{1} \in$ $\operatorname{nil}(\mathscr{H})$ for all $E_{1} \in \mathscr{H}_{1}$.

We shall prove that $\Omega \subset \mathscr{H}_{1}$. First we make the following preparatory remarks.

Consider $\mathscr{H}_{[0]}$-module $\mathscr{H}_{[-1]}$, and denote by $\rho$ the corresponding representation, that is, $\rho(E)=\left.(\operatorname{ad} E)\right|_{\mathscr{K}_{1-1}}, E \in \mathscr{H}_{[0\}}$. Fix the $\mathbb{F}$-basis $\left\{D_{1}, \ldots, D_{2 m}\right\}$ of $\mathscr{H}_{[-1]}$. For $E \in \mathscr{H}_{[0]}$, we identify $\rho(E)$ with its matrix with respect to the fixed basis. Let $\mathrm{pl}(m, m)$ denote the general linear Lie superalgebra of $2 m \times 2 m$ matrices over $\mathbb{F}$ (see [15]). Let

$$
\tilde{\mathrm{p}}(m)=\left\{\left[\begin{array}{cc}
A & B \\
C & -A^{\mathrm{T}}
\end{array}\right] \in \operatorname{pl}(m, m) \mid B=B^{\mathrm{T}}, C=-C^{\mathrm{T}}\right\} .
$$

Then $\tilde{\mathrm{p}}(m)$ is a subalgebra of $\mathrm{pl}(m, m)$ (see [8, page 16]).

In the following $e_{i j}$ denotes the $2 m \times 2 m$ matrix having 1 in $(i, j)$ position and 0 's elsewhere. The following lemma only needs straightforward verifications, which are omitted. 
LEMMA 2.6. The following statements hold:

(i) $\mathrm{T}_{\mathrm{H}}\left(x_{i^{\prime}} x_{j}\right)=(-1)^{\mu\left(i^{\prime}\right)+\mu\left(i^{\prime}\right) \mu(j)} x_{j} D_{i}+(-1)^{\mu(j)} x_{i^{\prime}} D_{j^{\prime}}, i, j \in Y$.

(ii) $\rho\left(\mathrm{T}_{\mathrm{H}}\left(x_{i^{\prime}} x_{j}\right)\right)=(-1)^{\mu(i)} e_{i j}-(-1)^{\mu(i) \mu(j)} e_{j^{\prime} i^{\prime}}, i, j \in Y$.

(iii) $\rho$ is faithful.

(iv) $\operatorname{Im}(\rho)=\tilde{\mathrm{p}}(m)$.

(v) If $E \in \operatorname{nil}\left(\mathscr{H}_{[0]}\right)$ then $\rho(E)$ is a nilpotent matrix.

THEOREM 2.7. Suppose that $E \in \operatorname{nil}\left(\mathscr{H}_{0}\right)$ and ad $E(\mathscr{H}) \subset \operatorname{nil}(\mathscr{H})$. Then $E \in \mathscr{H}_{1}$, that is, $\Omega \subset \mathscr{H}_{1} \cap \mathscr{H}_{\overline{0}}$.

PROOF. Decompose $E=E_{[-1]}+E_{0}$, where $E_{[-1]} \in \mathscr{H}_{[-1]} \cap \mathscr{H}_{0}, E_{0} \in \mathscr{H}_{0}$. Let $E_{[-1]}=\sum_{i \in \mathrm{Y}_{0}} c_{i} \mathrm{~T}_{\mathrm{H}}\left(x_{i^{\prime}}\right), c_{i} \in \mathbb{F}$. Assume that $E_{[-1]} \neq 0$. Without loss of generality we may assume that $c_{1}=1$. Applying (2), we obtain

$$
\left[E_{[-1]}, \mathrm{T}_{\mathrm{H}}\left(x^{\left(2 \varepsilon_{1}\right)} x_{1^{\prime}}\right)\right]=-\mathrm{T}_{\mathrm{H}}\left(x_{1} x_{1^{\prime}}\right) \text {. }
$$

By virtue of Lemma 2.4 and the equation above, we get $\left[E_{\{-1\}}, \mathrm{T}_{\mathrm{H}}\left(x^{\left(2 \varepsilon_{1}\right)} x_{1^{\prime}}\right)\right] \notin \operatorname{nil}(\mathscr{H})$. Now Lemma 2.2 shows $\left[E, \mathrm{~T}_{\mathrm{H}}\left(x^{\left(2 \varepsilon_{1}\right)} x_{1^{\prime}}\right)\right] \notin \operatorname{nil}(\mathscr{H})$, contradicting the assumption. Hence $E_{[-1]}=0, E=E_{0} \in \mathscr{H}_{0}$.

Assume that $E=E_{[0]}+E_{1}$, where $E_{[0]} \in \mathscr{H}_{[0]} \cap \mathscr{H}_{0}, E_{1} \in \mathscr{H}_{1} \cap \mathscr{H}_{0}$. By

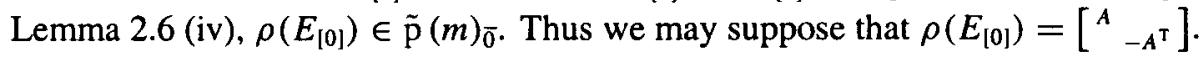

Assume that $E_{[0]} \neq 0$. According to Lemma 2.6 (iii), $A$ is a nonzero matrix. Put $A=\left(c_{i j}\right)_{m \times m}$. Suppose that the $l$-th row is the leading nonzero row and the $t$-th column is the leading nonzero column.

We treat two cases separately.

Case (i): $l \leq t$.

Let $k=\max \left\{j \in Y_{0} \mid c_{l j} \neq 0\right\}$. Then $l \leq t \leq k$.

Assume that $l=k$. Then $l=t=k$ and $c_{l l} \neq 0$. Obviously, $A$ is of the following block form $A=\left[\begin{array}{c}A_{l l} \\ * \\ *\end{array}\right]$, where $A_{l l}$ is an $l \times l$ matrix with $(l, l)$-entry $c_{l l} \neq 0$ and 0 elsewhere. So the matrix $\rho\left(E_{[0]}\right)$ is not nilpotent. By Lemma $2.6(\mathrm{v}), E_{[0]}$ is not adnilpotent. Then by Lemma 2.2, $E$ is not ad-nilpotent. This contradicts the assumption that $E \in \Omega \subset \operatorname{nil}(\mathscr{H})$. Thus $l<k$.

Obviously,

$$
\rho\left(E_{\{0\}}\right)=\sum_{j=t}^{k} c_{l j} e_{l j}+\sum_{i=l+1}^{m} \sum_{j=t}^{m} c_{i j} e_{i j}-\sum_{j=t}^{k} c_{l j} e_{j^{\prime} l^{\prime}}-\sum_{i=l+1}^{m} \sum_{j=t}^{m} c_{i j} c_{j^{\prime} i^{\prime}} .
$$

Direct computation shows that

$$
\begin{aligned}
& {\left[\rho\left(E_{[0]}\right), e_{k l}-e_{l^{\prime} k^{\prime}}\right]} \\
& \quad=c_{l k} e_{l l}-\sum_{j=l}^{k} c_{l j} e_{k j}+\sum_{i=l+1}^{m} c_{i k} e_{i l}-c_{l k} e_{l^{\prime} l^{\prime}}+\sum_{j=t}^{k} c_{l j} e_{j^{\prime} k^{\prime}}-\sum_{i=l+1}^{m} c_{i k} e_{l^{\prime} i^{\prime}} .
\end{aligned}
$$


This matrix possesses the block form $\left[\begin{array}{c}B_{l} \\ *_{*}\end{array}\right]$, where $B_{l l}$ is an $l \times l$ matrix in which $(l, l)$ element is $c_{l k} \neq 0$ and the others are all 0 . Therefore, the matrix $\left[\rho\left(E_{[0]}\right), e_{k l}-e_{l^{\prime} k^{\prime}}\right]$ is not nilpotent. By Lemma 2.6 (ii), $e_{k l}-e_{l^{\prime} k^{\prime}}=\rho\left(\mathrm{T}_{\mathrm{H}}\left(x_{k^{\prime}} x_{l}\right)\right)$, and the matrix $\rho\left(\left[\left(E_{[0]}, \mathrm{T}_{\mathrm{H}}\left(x_{k^{\prime}} x_{l}\right)\right]\right)\right.$ is not nilpotent. In combination with Lemma $2.6(\mathrm{v})$, we see that $\left[E_{[0]}, \mathrm{T}_{\mathrm{H}}\left(x_{k^{\prime}} x_{l}\right)\right]$ is not ad-nilpotent. Now Lemma 2.2 ensures that $\left[E, \mathrm{~T}_{\mathrm{H}}\left(x_{k^{\prime}} x_{l}\right)\right] \notin$ nil $(\mathscr{H})$. This contradicts the assumption that $E \in \Omega$.

Case (ii): $l>t$.

Let $k=\max \left\{i \in Y_{0} \mid c_{i t} \neq 0\right\}$. Then $k \geq l>t, a_{k t} \neq 0$ and

$$
\rho\left(E_{[0]}\right)=\sum_{i=l}^{k} c_{i t} e_{i t}+\sum_{j=t+1}^{m} \sum_{i=l}^{m} c_{i j} e_{i j}-\sum_{i=l}^{k} c_{i t} e_{t^{\prime} i^{\prime}}-\sum_{j=t+1}^{m} \sum_{i=l}^{m} c_{i j} e_{j^{\prime} i^{\prime}} .
$$

By Lemma 2.6 (ii), $\rho\left(\mathrm{T}_{\mathrm{H}}\left(x_{t^{\prime}} x_{k}\right)\right)=e_{t k}-e_{k^{\prime} t^{\prime}}$. Thus

$$
\begin{aligned}
& {\left[\rho\left(E_{[0]}\right), \rho\left(\mathrm{T}_{\mathrm{H}}\left(x_{t^{\prime}} x_{k}\right)\right)\right]} \\
& \quad=\sum_{i=l}^{k} c_{i t} e_{i k}-c_{k t} e_{t \prime}-\sum_{j=t+1}^{m} c_{k j} e_{t j}-\sum_{i=l}^{k} c_{i t} e_{k^{\prime} i^{\prime}}+c_{k t} e_{t^{\prime} t^{\prime}}+\sum_{j=t+1}^{m} c_{k j} e_{j^{\prime} t^{\prime}} .
\end{aligned}
$$

This matrix is of the following form $\left[\begin{array}{c}A_{1} \\ 0\end{array}\right]$, where $A_{t}$ is a $t \times t$ matrix whose $(t, t)$ entry is $-c_{k t} \neq 0$ and remaining entries are 0 . Proceeding analogously to Case (i), we may prove that $\left[E, \mathrm{~T}_{\mathbf{H}}\left(x_{t^{\prime}} x_{k}\right)\right]$ is not ad-nilpotent, contradicting the assumption that $E \in \Omega$.

We conclude that $E_{\{0 \mid}=0, E=E_{1} \in \mathscr{H}_{1}$.

\section{Natural filtration and classification}

For the sake of simplicity, an isomorphism between two odd Hamiltonian superalgebras will be called an $f$-isomorphism. In this section, we shall prove that the natural filtration of $\mathscr{H}$ is invariant under $f$-isomorphisms, that is, if $\varphi: \mathscr{H}(m ; t) \rightarrow \mathscr{H}\left(m^{\prime} ; t^{\prime}\right)$ is an isomorphism of Lie superalgebras, then $\varphi\left(\mathscr{H}(m ; \underline{t})_{i}\right)=\mathscr{H}\left(m^{\prime} ; \underline{t}^{\prime}\right)_{i}$ for all $i \geq-1$, where $m, m^{\prime} \in \mathbb{N} \backslash\{1,2\}, \underline{t} \in \mathbb{N}^{m}, \underline{t^{\prime}} \in \mathbb{N}^{m^{\prime}}$.

Lemma 3.1. Let $k, l \in Y_{0}$. Then $\mathrm{T}_{\mathrm{H}}\left(x^{\left(2 \varepsilon_{k}\right)} x_{l^{\prime}}\right) \in \Omega$ if and only if $k \neq l$.

Proof. Assume that $k=l$. By (2), $\left[\mathrm{T}_{\mathrm{H}}\left(x_{k^{\prime}}\right), \mathrm{T}_{\mathrm{H}}\left(x^{\left(2 \varepsilon_{k}\right)} x_{k^{\prime}}\right)\right]=-\mathrm{T}_{\mathrm{H}}\left(x_{k} x_{k^{\prime}}\right)$. By Lemma 2.4, we have $\mathrm{T}_{\mathrm{H}}\left(x_{k} x_{k^{\prime}}\right) \in \operatorname{nil}(\mathscr{H})$. Therefore, $\mathrm{T}_{\mathrm{H}}\left(x^{\left(2 \varepsilon_{k}\right)} x_{k^{\prime}}\right) \notin \Omega$.

Conversely, let $k \neq l$. Let $E=E_{[-1]}+E_{0}$ be an element of $\mathscr{H}$, where $E_{[-1]} \in$ $\mathscr{H}_{[-1]}, E_{0} \in \mathscr{H}_{0}$. Assume that $E_{[-1]}=\sum_{i \in Y} c_{i} \mathrm{~T}_{\mathrm{H}}\left(x_{i}\right)$, where $c_{i} \in \mathbb{F}$. Put $D:=$ $\left[E_{[-1]}, T_{H}\left(x^{\left(2 \varepsilon_{k}\right)} x_{l^{\prime}}\right)\right]$. Then

$$
D=\left[c_{k^{\prime}} \mathrm{T}_{\mathrm{H}}\left(x_{k^{\prime}}\right)+c_{l} \mathrm{~T}_{\mathrm{H}}\left(x_{l}\right), \mathrm{T}_{\mathrm{H}}\left(x^{\left(2 \varepsilon_{k}\right)} x_{l^{\prime}}\right)\right]=-c_{k^{\prime}} \mathrm{T}_{\mathrm{H}}\left(x_{k} x_{l^{\prime}}\right)+c_{l} \mathrm{~T}_{\mathrm{H}}\left(x^{\left(2 \varepsilon_{k}\right)}\right) .
$$


By Lemma 2.4, $\mathrm{T}_{\mathrm{H}}\left(x_{k} x_{l^{\prime}}\right)$ and $\mathrm{T}_{\mathrm{H}}\left(x^{\left(2 \varepsilon_{k}\right)}\right)$ are all ad-nilpotent elements. Applying (2), we obtain that $\left[\mathrm{T}_{\mathrm{H}}\left(x_{k} x_{l^{\prime}}\right), \mathrm{T}_{\mathrm{H}}\left(x^{\left(2 \varepsilon_{k}\right)}\right)\right]=0$. So $S:=\left\{0, \mathrm{~T}_{\mathrm{H}}\left(x_{k} x_{l^{\prime}}\right), \mathrm{T}_{\mathrm{H}}\left(x^{\left(2 \varepsilon_{k}\right)}\right)\right\}$ is a Lie subset of $\mathscr{H}$. By Lemma 2.1 and (4), we have $D \in \operatorname{nil}(\mathscr{H})$. Obviously,

$$
\left[E, \mathrm{~T}_{\mathrm{H}}\left(x^{\left(2 \varepsilon_{k}\right)} x_{l^{\prime}}\right)\right]=D+\left[E_{0}, \mathrm{~T}_{\mathrm{H}}\left(x^{\left(2 \varepsilon_{k}\right)} x_{l^{\prime}}\right)\right],
$$

where $\left[E_{0}, \mathrm{~T}_{\mathrm{H}}\left(x^{\left(2 \varepsilon_{k}\right)} x_{l^{\prime}}\right)\right] \in \mathscr{H}_{1}$. Note that $k \neq l$. It is easy to verify that $[D, D]=0$. By virtue of Lemma 2.5 and (5), we get $\left[E, \mathrm{~T}_{\mathrm{H}}\left(x^{\left(2 \varepsilon_{k}\right)} x_{l^{\prime}}\right)\right] \in \operatorname{nil}(\mathscr{H})$. Hence $\mathrm{T}_{\mathrm{H}}\left(x^{\left(2 \varepsilon_{k}\right)} x_{l^{\prime}}\right) \in \Omega$.

PROPOSITION 3.2. $\mathscr{H}_{1} \cap \mathscr{H}_{\overline{0}}=\Gamma$.

PRoOF. It is clear that $\mathscr{H}_{1} \cap \mathscr{H}_{\overline{0}} \subset \operatorname{nil}\left(\mathscr{H}_{\overline{0}}\right)$. By Theorem 2.7, $\Omega \subset \mathscr{H}_{1} \cap \mathscr{H}_{\overline{0}}$ and therefore, $\left[\mathscr{H}_{1} \cap \mathscr{H}_{\overline{0}}, \Omega\right] \subset\left[\mathscr{H}_{1} \cap \mathscr{H}_{\overline{0}}, \mathscr{H}_{1} \cap \mathscr{H}_{\overline{0}}\right] \subset \mathscr{H}_{2} \cap \mathscr{H}_{\overline{0}} \subset \Omega$. Thus $\mathscr{H}_{1} \cap \mathscr{H}_{0} \subset \Gamma$.

To prove the converse inclusion, we suppose that $E \in \Gamma$ and decompose $E=$ $E_{[-1]}+E_{0}$, where $E_{[-1]} \in \mathscr{H}_{[-1]}, E_{0} \in \mathscr{H}_{0}$. Assume that $E_{[-1]} \neq 0$. Since $E_{[-1]} \in \mathscr{H}_{\overline{0}}$, without loss of generality, we may suppose that $E_{[-1]}=D_{1}+\sum_{j=2}^{m} c_{j} D_{j}$, where $c_{j} \in \mathbb{F}$. Direct computation and application of Theorem 2.7 show that

$$
\left[E, \mathrm{~T}_{\mathrm{H}}\left(x^{\left(2 \varepsilon_{1}\right)} x_{2^{\prime}}\right)\right]=\mathrm{T}_{\mathrm{H}}\left(x_{1} x_{2^{\prime}}\right)+\left[E_{0}, \mathrm{~T}_{\mathrm{H}}\left(x^{\left(2 \varepsilon_{1}\right)} x_{2^{\prime}}\right)\right] \notin \Omega .
$$

By Lemma 3.1, $\mathrm{T}_{\mathrm{H}}\left(x^{\left(2 \varepsilon_{1}\right)} x_{2^{\prime}}\right) \in \Omega$. Moreover, (6) implies that $E \notin \Gamma$, which is a contradiction. So $E_{[-1]}=0, E=E_{0} \in \mathscr{H}_{0}$.

We next decompose $E_{0}=E=E_{[0]}+E_{1}$, where $E_{[0]} \in \mathscr{H}_{[0]}, E_{1} \in \mathscr{H}_{1}$. Assume that $E_{[0]} \neq 0$. Since $E_{[0]} \in \mathscr{H}_{0}$, we may assume that $E_{[0]}=\sum_{i, j \in Y_{0}} c_{i j} \mathrm{~T}_{\mathrm{H}}\left(x_{i} x_{j^{\prime}}\right)$, where $c_{i j} \in \mathbb{F}$. Put

$$
\begin{aligned}
& l:=\min \left\{i \in Y_{0} \mid c_{i j_{0}} \neq 0 \text { for some } j_{0} \in Y\right\} \\
& t:=\min \left\{j \in Y_{0} \mid c_{i_{0}} \neq 0 \text { for some } i_{0} \in Y\right\} .
\end{aligned}
$$

Case (i): $l \leq t$.

Let $k:=\max \left\{j \in Y_{0} \mid c_{l j} \neq 0\right\}$. Then $l \leq t \leq k$ and $c_{l k} \neq 0$.

If $l=k$, proceeding similarly as in the proof of Theorem 2.7 , we may prove that $E$ is not ad-nilpotent, which gives a contradiction.

If $l<k$, then

$$
E_{[0]}=\sum_{j=t}^{k} c_{l j} \mathrm{~T}_{\mathrm{H}}\left(x_{l} x_{j^{\prime}}\right)+\sum_{j=l+1}^{m} \sum_{j=t}^{m} c_{i j} \mathrm{~T}_{\mathrm{H}}\left(x_{i} x_{j^{\prime}}\right)
$$


Let $D:=\left[\mathrm{T}_{\mathrm{H}}\left(x^{\left(2 \varepsilon_{k}\right)} x_{l^{\prime}}\right), E_{[0]}\right]$. Then

$$
\begin{aligned}
D & =\left[x_{k} x_{l^{\prime}} D_{k^{\prime}}-x^{\left(2 \varepsilon_{k}\right)} D_{l}, E_{[0]}\right] \\
& =c_{l k} \mathrm{~T}_{\mathrm{H}}\left(x_{k} x_{l^{\prime}} x_{l}\right)-\sum_{j=t}^{k} c_{l j} \mathrm{~T}_{\mathrm{H}}\left(x^{\left(2 \varepsilon_{k}\right)} x_{j^{\prime}}\right)+\sum_{j=l+1}^{m} c_{i k} \mathrm{~T}_{\mathrm{H}}\left(x_{k} x_{l^{\prime}} x_{i}\right) .
\end{aligned}
$$

Therefore,

$$
\left[\mathrm{T}_{\mathrm{H}}\left(x_{k^{\prime}}\right), D\right]=-c_{l k} \mathrm{~T}_{\mathrm{H}}\left(x_{l^{\prime}} x_{l}\right)+\sum_{j=\imath}^{k} c_{l j} \mathrm{~T}_{\mathrm{H}}\left(x_{k} x_{j^{\prime}}\right)-\sum_{j=l+1}^{m} c_{i k} \mathrm{~T}_{\mathrm{H}}\left(x_{l^{\prime}} x_{i}\right) .
$$

By Lemma 2.6 (ii), we have

$$
\rho\left(\left[\mathrm{T}_{\mathrm{H}}\left(x_{k^{\prime}}\right), D\right]\right)=-c_{l k}\left(e_{l l}-e_{l^{\prime} l^{\prime}}\right)+\sum_{j=t}^{k} c_{l j}\left(e_{j k}-e_{k^{\prime} j^{\prime}}\right)-\sum_{j=l+1}^{m} c_{i k}\left(e_{l i}-e_{i^{\prime} l^{\prime}}\right) .
$$

This matrix is of the following block form $\left[\begin{array}{c}A_{l l}^{*} \\ 0\end{array}\right]$, where $A_{l l}$ is an $l \times l$ matrix whose $(l, l)$-entry is $-c_{l k} \neq 0$, but other entries are 0 . Consequently, the matrix $\rho\left(\left[\mathrm{T}_{\mathrm{H}}\left(x_{k^{\prime}}\right), D\right]\right)$ is not nilpotent. This and Lemma $2.6(\mathrm{v})$ show that $\left[\mathrm{T}_{\mathrm{H}}\left(x_{k^{\prime}}\right), D\right]$ is not ad-nilpotent. By Lemma 2.2, $\left[\mathrm{T}_{\mathrm{H}}\left(x_{k^{\prime}}\right),\left[\mathrm{T}_{\mathrm{H}}\left(x^{\left(2 \varepsilon_{k}\right)} x_{l^{\prime}}\right), E\right]\right]$ is not ad-nilpotent. Furthermore, we obtain that

$$
\left[\mathrm{T}_{\mathrm{H}}\left(x^{\left(2 \varepsilon_{k}\right)} x_{l^{\prime}}\right), E\right] \notin \Omega .
$$

On the other hand, by Lemma 3.1, $\mathrm{T}_{\mathrm{H}}\left(x^{\left(2 \varepsilon_{k}\right)} x_{l^{\prime}}\right) \in \Omega$. Hence (7) implies that $E \notin \Gamma$, which is a contradiction.

Case (ii): $l>t$.

Let $k:=\max \left\{i \in Y_{0} \mid c_{i t} \neq 0\right\}$. Then $k \geq l>t, c_{k t} \neq 0$ and

$$
E_{[0]}=\sum_{i=l}^{k} c_{i t} \mathrm{~T}_{\mathrm{H}}\left(x_{i} x_{t^{\prime}}\right)+\sum_{i=l}^{m} \sum_{j=t+1}^{m} c_{i j} \mathrm{~T}_{\mathrm{H}}\left(x_{i} x_{j^{\prime}}\right)
$$

Put $G:=\left[\mathrm{T}_{\mathrm{H}}\left(x^{\left(2 \varepsilon_{t}\right)} x_{k^{\prime}}\right), E_{[0]}\right]$. Using (2) we compute

$$
G=\sum_{i=l}^{k} c_{i t} \mathrm{~T}_{\mathrm{H}}\left(x_{t} x_{k^{\prime}} x_{i}\right)-c_{k t} \mathrm{~T}_{\mathrm{H}}\left(x^{\left(2 \varepsilon_{i}\right)} x_{t^{\prime}}\right)-\sum_{j=t+1}^{m} c_{k j} \mathrm{~T}_{\mathrm{H}}\left(x^{\left(2 \varepsilon_{i}\right)} x_{j^{\prime}}\right)
$$

Therefore,

$$
\left[\mathrm{T}_{H}\left(x_{t^{\prime}}\right), G\right]=c_{k t} \mathrm{~T}_{H}\left(x_{t} x_{t^{\prime}}\right)-\sum_{i=l}^{k} c_{i t} \mathrm{~T}_{H}\left(x_{k^{\prime}} x_{i}\right)+\sum_{j=t+1}^{m} c_{k j} \mathrm{~T}_{H}\left(x_{i} x_{j^{\prime}}\right) .
$$


By Lemma 2.6 (ii),

$$
\rho\left(\left[\mathrm{T}_{\mathrm{H}}\left(x_{t^{\prime}}\right), G\right]\right)=c_{k t}\left(e_{t t}-e_{t^{\prime} t^{\prime}}\right)-\sum_{i=l}^{k} c_{i t}\left(e_{k i}-e_{i^{\prime} k^{\prime}}\right)+\sum_{j=t+1}^{m} c_{k j}\left(e_{j t}-e_{t^{\prime} j^{\prime}}\right) .
$$

This matrix is of the form $\left[\begin{array}{c}B_{l} \\ *_{*}^{*}\end{array}\right]$, where $B_{l l}$ is an $l \times l$ matrix whose $(l, l)$-entry is $c_{k t} \neq 0$, but other entries are 0 . Similar to (i), we obtain that $\left[\mathrm{T}_{\mathrm{H}}\left(x^{\left(2 \varepsilon_{1}\right)} x_{k^{\prime}}\right), E\right] \notin \Omega$. By Lemma 3.1, $\mathrm{T}_{\mathrm{H}}\left(x^{\left(2 \varepsilon_{1}\right)} x_{k^{\prime}}\right) \in \Omega$ and therefore $E \notin \Gamma$, a contradiction.

Combining (i) and (ii), we conclude that $E_{[0]}=0$ and $E=E_{1} \in \mathscr{H}_{1}$. This proves that $\Gamma \subset \mathscr{H}_{1} \cap \mathscr{H}_{\overline{0}}$.

PROPOSITION 3.3. $\mathscr{H}_{0}=\Phi$.

PROOF. The inclusion $\mathscr{H}_{0} \subset \Phi$ is clear. So, we need only to prove the converse inclusion. Assume that $E=E_{[-1]}+E_{0} \in \Phi$, where $E_{[-1]} \in \mathscr{H}_{[-1]}, E_{0} \in \mathscr{H}_{0}$. Let $E_{[-1]}=\sum_{i \in Y} c_{i} \mathrm{~T}_{\mathbf{H}}\left(x_{i}\right), c_{i} \in \mathbb{F}$. Assume that $E_{[-1]} \neq 0$. Then there exists some $k \in Y$ such that $c_{k} \neq 0$. If $k \in Y_{1}$, we may let $k=1^{\prime}$. Put $D:=\left[E_{[-1]}, \mathbf{T}_{\mathrm{H}}\left(x^{\left(2 \varepsilon_{1}\right)} x_{1^{\prime}}\right)\right]$. Then we have

$$
\begin{aligned}
D & =\left[c_{1} \mathrm{~T}_{\mathrm{H}}\left(x_{1}\right)+c_{1^{\prime}} \mathrm{T}_{\mathrm{H}}\left(x_{1^{\prime}}\right), \mathrm{T}_{\mathrm{H}}\left(x^{\left(2 \varepsilon_{1}\right)} x_{1^{\prime}}\right)\right] \\
& =c_{1} \mathrm{~T}_{\mathrm{H}}\left(x^{\left(2 \varepsilon_{1}\right)}\right)-c_{1^{\prime}} \mathrm{T}_{\mathrm{H}}\left(x_{1} x_{1^{\prime}}\right) \\
& =c_{1} x_{1} D_{1^{\prime}}-c_{1^{\prime}}\left(x_{1^{\prime}} D_{1^{\prime}}-x_{1} D_{1}\right) .
\end{aligned}
$$

Therefore, $D^{l}\left(x_{1}\right)=c_{1}^{l}, x_{1}$ for all $l \in \mathbb{N}$. Thus $D$ is not nilpotent as a linear transformation. By Lemma 2.3, $D$ is not ad-nilpotent. Now Lemma 2.2 shows that $\left[E, \mathrm{~T}_{\mathrm{H}}\left(x^{\left(2 \varepsilon_{1}\right)} x_{1^{\prime}}\right)\right]$ is not ad-nilpotent. Observe that $\mathrm{T}_{\mathrm{H}}\left(x^{\left(2 \varepsilon_{1}\right)} x_{1^{\prime}}\right) \in \mathscr{H}_{1} \cap \mathscr{H}_{\overline{0}}$. This contradicts the assumption that $E \in \Phi$. Hence $E_{[-1]}=\sum_{i \in \mathrm{Y}_{0}} c_{i} \mathrm{~T}_{\mathrm{H}}\left(x_{i}\right)$. Without loss of generality, we may suppose that $c_{1} \neq 0$. Let $G:=\mathrm{T}_{\mathrm{H}}\left(x_{1}, x_{2} x_{3}+x_{1^{\prime}} x_{2^{\prime}} x_{3^{\prime}}\right)$. Then

$$
\left[E_{[-1]}, G\right]=c_{1} \mathrm{~T}_{\mathrm{H}}\left(x_{2} x_{3}+x_{2^{\prime}}, x_{3^{\prime}}\right)-c_{2} \mathrm{~T}_{\mathbf{H}}\left(x_{1^{\prime}} x_{3^{\prime}}\right)+c_{3} \mathrm{~T}_{\mathbf{H}}\left(x_{1^{\prime}}, x_{2^{\prime}}\right) .
$$

Therefore,

$$
\left(\operatorname{ad}\left[E_{[-1]}, G\right]\right)^{4 t}\left(\mathrm{~T}_{\mathrm{H}}\left(x_{2}+x_{3}\right)\right)=c_{1}^{4 t} \mathrm{~T}_{\mathrm{H}}\left(x_{2}+x_{3}\right) \quad \text { for all } t \in \mathbb{N} .
$$

By Lemma 2.2, $[E, G] \notin \operatorname{nil}(\mathscr{H})$. Notice that $G \in \mathscr{H}_{1} \cap \mathscr{H}_{0}$. This contradicts the assumption that $E \in \Phi$. Hence $E_{[-1]}=0, E \in \mathscr{H}_{0}$. So $\Phi \subset \mathscr{H}_{0}$, as required.

Before proving the following main theorem we recall the notation introduced in the beginning of Section 2.

THEOREM 3.4. The natural filtrations of finite-dimensional odd Hamiltonian superalgebras are invariant under f-isomorphisms. 
Proof. Let $m, m^{\prime} \in \mathbb{N} \backslash\{1,2\}, \underline{t} \in \mathbb{N}^{m}, \underline{t}^{\prime} \in \mathbb{N}^{m^{\prime}}$ and $\varphi: \mathscr{H}(m ; \underline{t}) \rightarrow \mathscr{H}\left(m^{\prime} ; \underline{t}^{\prime}\right)$ be an $f$-isomorphism. Observe that $\varphi$ preserves $\mathbb{Z}_{2}$-gradations. By the definition of $\Omega$, it is clear that $\varphi(\Omega)=\Omega^{\prime}$; furthermore, $\varphi(\Gamma)=\Gamma^{\prime}$. By Proposition 3.2 and the definition of $\Phi, \varphi(\Phi)=\Phi^{\prime}$. This and Proposition 3.3 ensure that $\varphi\left(\mathscr{H}(m ; t)_{0}\right)=\mathscr{H}\left(m^{\prime} ; \underline{t}^{\prime}\right)_{0}$.

As

$$
\mathscr{H}_{i}=\left\{E \in \mathscr{H}_{i-1} \mid \text { ad } E(\mathscr{H}) \subset \mathscr{H}_{i-1}\right\}, \quad i \geq 1,
$$

we may prove, by induction on $i$, that $\varphi\left(\mathscr{H}(m ; t)_{i}\right)=\mathscr{H}\left(m^{\prime} ; \underline{t}^{\prime}\right)_{i}$ for all $i \geq-1$.

COROLLARY 3.5. The filtration of finite-dimensional odd Hamiltonian superalgebra $\mathscr{H}$ is invariant under Aut $\mathscr{H}$.

ProOF. This is a direct consequence of Theorem 3.4.

As a direct application of Theorem 3.4, we establish the following property of isomorphisms of odd Hamiltonian superalgebras.

By Theorem 3.4, we may easily prove the following

COROLlaRY 3.6. Let $\phi$ and $\varphi$ be f-isomorphisms of $\mathscr{H}(m ; t)$ to $\mathscr{H}\left(m^{\prime} ; t^{\prime}\right)$. Then $\phi=\varphi$ if and only if $\left.\phi\right|_{\mathscr{H}_{1-11}}=\left.\varphi\right|_{\mathscr{K}_{[-11}}$.

Employing Theorem 3.4, we may prove that $m$ and $\underline{t}$ are intrinsic for the odd Hamiltonian superalgebra $\mathscr{H}(m ; t)$, that is, we may give a classification of odd Hamiltonian superalgebras. For $\underline{t}, \underline{t^{\prime}} \in \mathbb{N}^{m}, \underline{t}, \underline{t^{\prime}}$ are said to be equivalent and denoted by $\underline{t} \sim \underline{t}^{\prime}$ if there exists a permutation $\sigma \in S_{m}$ such that $t_{\sigma(i)}=t_{i}^{\prime}$ for all $i \in Y_{0}$.

THEOREM 3.7. Suppose that $m, m^{\prime} \in \mathbb{N} \backslash\{1,2\}, \underline{t} \in \mathbb{N}^{m}, \underline{t}^{\prime} \in \mathbb{N}^{m^{\prime}}$. Then $\mathscr{H}(m ; \underline{t} \cong$ $\mathscr{H}\left(m^{\prime} ; \underline{t}^{\prime}\right)$ if and only if $m=m^{\prime}$ and $\underline{t} \sim \underline{t}^{\prime}$.

Proof. Assume that $\phi: \mathscr{H}(m ; \underline{t}) \rightarrow \mathscr{H}\left(m^{\prime} ; \underline{t}^{\prime}\right)$ is an isomorphism of Lie superalgebras. Then Theorem 3.4 ensures that $\phi$ induces canonically an isomorphism of quotient spaces: $\mathscr{H}(m ; \underline{t}) / \mathscr{H}(m ; \underline{t})_{0} \rightarrow \mathscr{H}\left(m^{\prime} ; \underline{t^{\prime}}\right) / \mathscr{H}\left(m^{\prime} ; \underline{t^{\prime}}\right)_{0}$. Note that

$$
\operatorname{dim}\left(\mathscr{H}(m ; \underline{t}) / \mathscr{H}(m ; \underline{t})_{0}\right)=\operatorname{dim} \mathscr{H}(m ; t)_{[-1]}=2 m .
$$

It follows that $m=m^{\prime}$.

Without loss of generality, we may suppose that $t_{1} \geq \cdots \geq t_{m}$ and $t_{1}^{\prime} \geq \cdots \geq t_{m}^{\prime}$. Assume on the contrary that $\underline{t} \neq \underline{t}^{\prime}$. Then we may suppose that for some $k \in Y_{0}$,

$$
t_{k}>t_{k}^{\prime} \text { but } t_{j}=t_{j}^{\prime} \text { for } k<j \leq m \text { (maybe } k=m \text { ). }
$$


We assert that $\mathscr{H}(m ; \underline{t})_{\left[p^{\prime} t_{k}\right]} \supsetneq \mathscr{H}\left(m ; \underline{t}^{\prime}\right)_{\left[p^{\prime} t_{k}\right]^{\prime}}$. According to (8) and the definition of $\mathscr{H}(m ; t)$, the implication ' $\supset$ ' is clear. Notice that

$$
\mathrm{T}_{\mathrm{H}}\left(x^{\left(p^{\prime} \varepsilon_{k}\right)}\right) \in \mathscr{H}(m ; \underline{t})_{\left[p^{\prime \prime}-2\right]} \text { but } \quad \mathrm{T}_{\mathrm{H}}\left(x^{\left(p^{\left.p^{\prime} \varepsilon_{k}\right)}\right.}\right) \notin \mathscr{H}\left(m ; \underline{t}^{\prime}\right)_{\left[p_{k-2]}^{\prime}\right.} .
$$

So our assertion holds and therefore, $\operatorname{dim} \mathscr{H}\left(m ; t_{\left[p^{\left.p^{\prime}-2\right]}\right.}>\operatorname{dim} \mathscr{H}\left(m ; \underline{t}^{\prime}\right)_{\left[p^{\prime} x_{-2}\right]^{\circ}}\right.$ On the other hand, Theorem 3.4 implies that

$$
\phi\left(\mathscr{H}(m ; t)_{i}\right)=\mathscr{H}\left(m ; \underline{t}^{\prime}\right)_{i} \text { for all } i \geq-1 .
$$

From this we see easily that $\operatorname{dim} \mathscr{H}(m ; \underline{t})_{[i]}=\operatorname{dim} \mathscr{H}\left(m ; \underline{t}^{\prime}\right)_{[i]}$ for all $i \geq-1$. In particular, $\operatorname{dim} \mathscr{H}(m ; \underline{t})_{\left[p_{k}^{\left.t_{k}^{\prime}-2\right]}\right.}=\operatorname{dim} \mathscr{H}\left(m ; \underline{t}^{\prime}\right)_{\left[p^{\prime}-2\right]}$, contradicting to (9).

The converse implication is automatic. The proof is completed.

\section{The automorphism group of $\mathscr{H}(m, m ; \underline{1})$}

Recall that a Lie superalgebra $L=L_{\overline{0}} \oplus L_{\overline{\mathrm{T}}}$ over $\mathbb{F}$ is called restricted, if the Lie algebra $L_{\overline{0}}$ is restricted and the $L_{\overline{0}}$-module $L_{\overline{1}}$ is restricted (see [13]). The proof of Lemma 4.1 is analogous to [18, Theorem $4.4 .5(2)]$ or [23, Theorem 5].

LEMMA 4.1. $\mathscr{H}(m ; t)$ is restricted if and only if $\underline{t}=1$.

Let $\mathscr{A}$ be a finite-dimensional superalgebra over $\mathbb{F}$. Denote by Aut $\mathscr{A}$ the (even) automorphism group of $\mathscr{A}$. If $\sigma \in$ Aut $\mathscr{A}$ and $D \in \operatorname{Der} \mathscr{A}$, then $D^{\sigma}:=\sigma D \sigma^{-1}$ is again a superderivation of $\mathscr{A}$. It is easy to see that $\tilde{\sigma}: D \rightarrow D^{\sigma}$ is an automorphism of Der $\mathscr{A}$. Suppose that $\mathscr{Q}$ is a Lie subsuperalgebra of Der $\mathscr{A}$. We call $\sigma \in$ Aut $\mathscr{A}$ admissible to $\mathscr{Q}$ if $\tilde{\sigma}(\mathscr{Q}) \subset \mathscr{Q}$. Put $\operatorname{Aut}(\mathscr{A}: \mathscr{Q}):=\{\sigma \in$ Aut $\mathscr{A} \mid \tilde{\sigma}(\mathscr{Q}) \subset \mathscr{Q}\}$. Then Aut $(\mathscr{A}: \mathscr{Q})$ is a subgroup of Aut $\mathscr{A}$, and is referred to as the admissible automorphism group of $\mathscr{A}\left(\right.$ to $\mathscr{Q}$ ). Obviously, $\Phi: \operatorname{Aut}(\mathscr{A}: \mathscr{Q}) \rightarrow$ Aut $\mathscr{Q},\left.\sigma \mapsto \tilde{\sigma}\right|_{\mathscr{Q}}$ is a homomorphism of groups. In this section, we only deal with the restricted odd Hamiltonian superalgebra $\mathscr{H}(m ; \underline{1})$, and therefore adopt the convention $\mathscr{U}:=$ $\Lambda(m, m ; 1), \mathscr{H}:=\mathscr{H}(m ; 1)$ and $W:=W(m, m ; 1)$.

The main result of this section is the following theorem.

THEOREM 4.2. Let $\Phi: \operatorname{Aut}(\mathscr{U}: \mathscr{H}) \rightarrow$ Aut $\mathscr{H},\left.\sigma \mapsto \tilde{\sigma}\right|_{\mathscr{H}}$. Then $\Phi$ is an isomorphism of groups.

To prove it, we need the following lemmas. First we introduce some notation. Let $\mathrm{M}_{2 m}(\mathscr{U})$ denote the $\mathbb{F}$-algebra consisting of all $2 m \times 2 m$ matrices over $\mathscr{U}, \mathrm{pr}_{[0 \mid}$ and $\mathrm{pr}_{1}$ be the projections of $\mathscr{U}$ onto $\mathscr{U}_{[0]}=\mathbb{F}$ and $\mathscr{U}_{1}$, respectively. For $A=\left(a_{i j}\right) \in \mathrm{M}_{2 m}(\mathscr{U})$, set $\mathrm{pr}_{[0]} A:=\left(\mathrm{pr}_{[0]}\left(a_{i j}\right)\right)$ and $\mathrm{pr}_{1} A:=\left(\mathrm{pr}_{1}\left(a_{i j}\right)\right)$. 
LEMMA 4.3. The following statements hold:

(i) Let $A \in \mathrm{M}_{2 m}(\mathscr{U})$. Then $A$ is invertible if and only if $\mathrm{pr}_{[0]} A$ is invertible matrix over $\mathbb{F}$.

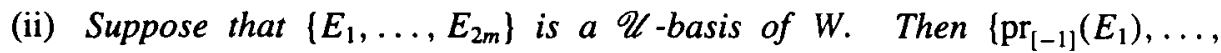
$\left.\operatorname{pr}_{[-1]}\left(E_{2 m}\right)\right\}$ is an $\mathbb{F}$-basis of $W_{[-1]}$, where $\operatorname{pr}_{[-1]}$ is the projection of $W$ onto $W_{[-1]}$.

(iii) Suppose that $\phi \in$ Aut $\mathscr{H}$ and $\left\{G_{i} \mid i \in Y\right\} \subset \mathscr{H}$ is a $\mathscr{U}$-basis of $W$. Then $\left\{\phi\left(G_{i}\right) \mid i \in Y\right\}$ is also a $\mathscr{U}$-basis of $W$.

Proof. (i) Clearly, $A=\operatorname{pr}_{[0]} A+\operatorname{pr}_{1} A$. Since every element of $\mathscr{U}_{1}$ is nilpotent, so is every $2 m \times 2 m$ matrix over $\mathscr{U}_{1}$. From these facts one may easily prove (i).

(ii) Suppose that $\left(D_{1}, \ldots, D_{2 m}\right)^{\mathrm{T}}=A\left(E_{1}, \ldots, E_{2 m}\right)^{\mathrm{T}}, A \in \mathrm{M}_{2 m}(\mathscr{U})$. Then $\left(D_{1}, \ldots, D_{2 m}\right)^{\mathrm{T}}=\left(\operatorname{pr}_{[0]} A\right)\left(\operatorname{pr}_{[-1]}\left(E_{1}\right), \ldots, \operatorname{pr}_{[-1]}\left(E_{2 m}\right)\right)^{\mathrm{T}}$. Since $\left\{D_{1}, \ldots, D_{2 m}\right\}$ is an $\mathbb{F}$-basis of $W_{[-1]}$, so is $\left\{\mathrm{pr}_{[-1]}\left(E_{1}\right), \ldots, \mathrm{pr}_{[-1]}\left(E_{2 m}\right)\right\}$.

(iii) By Corollary 3.5, the natural filtration $\left\{\mathscr{H}_{i}\right\}$ is invariant under $\phi$. Thus $\phi$ induces canonically $\bar{\phi} \in \mathrm{GL}\left(\mathscr{H} / \mathscr{H}_{0}\right)$. Denote by $\bar{G}_{i}$ the image of $G_{i}$ under the canonical map $\mathscr{H} \rightarrow \mathscr{H} / \mathscr{H}_{0}$. Then $\left\{\bar{G}_{i} \mid i \in Y\right\}$ is an $\mathbb{F}$-basis of $\mathscr{H} / \mathscr{H}_{0}$. Assume that

$$
\left(\phi\left(G_{1}\right), \ldots, \phi\left(G_{2 m}\right)\right)^{\mathrm{T}}=A\left(D_{1}, \ldots, D_{2 m}\right)^{\mathrm{T}}, \quad A \in \mathrm{M}_{2 m}(\mathscr{U}) .
$$

Decompose $A=\operatorname{pr}_{[0]} A+\operatorname{pr}_{1} A$. We obtain that

$$
\left(\bar{\phi}\left(\bar{G}_{1}\right), \ldots, \bar{\phi}\left(\bar{G}_{2 m}\right)\right)^{\mathrm{T}}=\left(\overline{\phi\left(G_{1}\right)}, \ldots, \overline{\phi\left(G_{2 m}\right)}\right)^{\mathrm{T}}=\left(\mathrm{pr}_{[0]} A\right)\left(\bar{D}_{1}, \ldots, \bar{D}_{2 m}\right)^{\mathrm{T}} .
$$

This implies that $\operatorname{pr}_{[0]} A$ is invertible. By (i), $A$ is invertible and therefore $\left\{\phi\left(G_{i}\right)\right\}$ $i \in Y\}$ is a $\mathscr{U}$-basis of $W$.

LEMMA 4.4. Suppose that $\phi \in$ Aut $\mathscr{H}$. Then there exist $y_{j} \in \mathscr{U}_{1}$ with $\operatorname{deg}\left(y_{j}\right)=$ $\mu(j)$ such that $\left(\phi\left(D_{i}\right)\right)\left(y_{j}\right)=\delta_{i j}+\delta_{j 1} \delta_{i 1}$ for $i, j \in Y$. In particular, the matrix $\left(\left(\phi\left(D_{i}\right)\right)\left(y_{j}\right)\right)_{i, j \in Y}$ is invertible.

Proof. Let $j \in Y$. By Lemma 4.3 (iii), $\left\{\phi\left(D_{1}\right), \ldots, \phi\left(D_{2 m}\right)\right\}$ is a $\mathscr{U}$-basis of $W$. Thus we may suppose that $\phi\left(\mathrm{T}_{\mathrm{H}}\left(x_{1} x_{j}\right)\right)=\sum_{l=1}^{2 m} a_{j l} \phi\left(D_{l}\right)$, where $a_{j l} \in \mathscr{U}$. From Lemma 4.3 (ii), we see easily that $a_{j l} \in \mathscr{U}_{1}$. Using (1), we obtain that

$$
\phi\left(\left[D_{i}, \mathrm{~T}_{\mathrm{H}}\left(x_{1} x_{j}\right)\right]\right)=\left[\phi\left(D_{i}\right), \sum_{l=1}^{2 m} a_{j l} \phi\left(D_{l}\right)\right]=\sum_{l=1}^{2 m}\left(\phi\left(D_{i}\right)\left(a_{j l}\right)\right) \phi\left(D_{l}\right) .
$$

On the other hand, by Lemma $2.6(\mathrm{i}), \mathrm{T}_{\mathrm{H}}\left(x_{1} x_{j}\right)=x_{j} D_{1^{\prime}}+(-1)^{\mu(j)} x_{1} D_{j^{\prime}}$ and therefore,

$$
\phi\left(\left[D_{i}, \mathrm{~T}_{\mathrm{H}}\left(x_{1} x_{j}\right)\right]\right)=\delta_{i j} \phi\left(D_{1^{\prime}}\right)+(-1)^{\mu(j)} \delta_{i 1} \phi\left(D_{j^{\prime}}\right) .
$$


Comparing (10) and (11), one gets $\phi\left(D_{i}\right)\left(a_{j 1}\right)=\delta_{i j}+\delta_{j 1} \delta_{i 1}$. Put $y_{j}:=a_{j 1^{\prime}}$ for $j \in Y$. We see that $\phi\left(D_{i}\right)\left(y_{j}\right)=\delta_{i j}+\delta_{j 1} \delta_{i 1}, y_{j} \in \mathscr{U}_{1}$ and $\operatorname{deg}\left(y_{j}\right)=\operatorname{deg}\left(a_{j 1^{\prime}}\right)=$ $\mu\left(j^{\prime}\right)+\mu\left(1^{\prime}\right)=\mu(j)$, as desired.

Proof of Theorem 4.2. Let $\sigma \in \operatorname{Aut}(\mathscr{U}: \mathscr{H})$. Assume that $\left.\tilde{\sigma}\right|_{\mathscr{H}}=\left.1\right|_{\mathscr{H}}$. We proceed by induction on $|\alpha|+|u|$ to show that $\sigma\left(x^{(\alpha)} x^{u}\right)=x^{(\alpha)} x^{u}$. Note that $W_{[-1]}=\mathscr{H}_{[-1]}$. We obtain that

$$
D_{j} x_{i}=\delta_{i j}=\sigma\left(\delta_{i j}\right)=\sigma\left(D_{j} x_{i}\right)=D_{j}^{\sigma}\left(\sigma\left(x_{i}\right)\right)=D_{j}\left(\sigma\left(x_{i}\right)\right), \quad i, j \in Y .
$$

This implies that $x_{i}-\sigma\left(x_{i}\right) \in \mathbb{F}$. Since $\sigma\left(\mathscr{U}_{1}\right) \subset \mathscr{U}_{1}$, it follows that $\sigma\left(x_{i}\right)=x_{i}$, $i \in Y$. Suppose that $|\alpha|+|u|>1$. Then by induction hypothesis, we obtain

$$
D_{i}\left(\sigma\left(x^{(\alpha)} x^{u}\right)-x^{(\alpha)} x^{u}\right)=\sigma\left(D_{i}\left(x^{(\alpha)} x^{u}\right)\right)-D_{i}\left(x^{(\alpha)} x^{u}\right)=0 \text { for all } i \in Y,
$$

and therefore $\sigma\left(x^{(\alpha)} x^{u}\right)-x^{(\alpha)} x^{u} \in \mathbb{F}$. Thus $\sigma\left(x^{(\alpha)} x^{u}\right)=x^{(\alpha)} x^{u}$. Consequently, $\sigma=1$ and $\Phi$ is injective.

We next prove that $\Phi$ is surjective. Let $\phi \in$ Aut $\mathscr{H}$. By Lemma 4.4 there exists $y_{j} \in \mathscr{U}$, with $\operatorname{deg}\left(y_{j}\right)=\mu(j)$ such that $\left(\phi\left(D_{i}\right)\right)\left(y_{j}\right)=\delta_{i j}+\delta_{j 1} \delta_{i 1}$. Assume that $\phi\left(D_{i}\right)=\sum_{j=1}^{2 m} a_{i j} D_{j}, a_{i j} \in \mathscr{U}$. Then we have the matrix equation $\left(\phi\left(D_{i}\right)\left(y_{j}\right)\right)=$ $\left(a_{i j}\right)\left(D_{i} y_{j}\right)$ and therefore,

$$
\left(\delta_{i j}+\delta_{j 1} \delta_{i 1}\right)=\left(\phi\left(D_{i}\right)\left(y_{j}\right)\right)=\operatorname{pr}_{[0]}\left(\phi\left(D_{i}\right)\left(y_{j}\right)\right)=\operatorname{pr}_{[0]}\left(c_{i j}\right) \operatorname{pr}_{[0\}}\left(D_{i} y_{j}\right) .
$$

Thus $\operatorname{pr}_{[0]}\left(D_{i} y_{j}\right)$ is invertible. Define the endomorphism $\sigma$ of $\mathscr{U}$ such that

$$
\sigma\left(x_{i}\right)=y_{j} \quad \text { for all } i \in Y .
$$

Then $\sigma$ is even. We claim that $\sigma \in$ Aut $\mathscr{U}$. From (12) it is easy to see that $\sigma$ leaves the natural filtration of $\mathscr{U}$ invariant, that is, $\sigma\left(\mathscr{U}_{i}\right) \subset \mathscr{U}_{i}$ for all $i \geq 0$. Therefore, it induces linear transformations $\sigma_{i}$ of $\mathscr{U}_{i} / \mathscr{U}_{i+1}, i \geq 0$. Note that the matrix of $\sigma_{1}$ relative to $\mathbb{F}$-basis $\left\{x_{1}+\mathscr{U}_{2}, \ldots, x_{2 m}+\mathscr{U}_{2}\right\}$ is just $\left(\operatorname{pr}_{[0]}\left(D_{i} y_{j}\right)\right)$. It follows that $\sigma_{1}$ is bijective. Proceeding by induction on $i \geq 1$, one may prove that $\sigma_{i}$ is bijective. Now our claim follows.

Note that $\tilde{\sigma}\left(D_{i}\right)\left(y_{j}\right)=\left(\sigma D_{i} \sigma^{-1}\right)\left(y_{j}\right)=\sigma\left(D_{i} x_{j}\right)=\delta_{i j}=\phi\left(D_{i}\right)\left(y_{j}\right)$ for all $i, j \in Y$. Since $\left\{y_{j} \mid j \in Y\right\}$ generates $\mathscr{U}$, we conclude that $\tilde{\sigma}\left(D_{i}\right)=\phi\left(D_{i}\right), i \in Y$. By induction on $k$, we may prove that $\left.\tilde{\sigma}\right|_{\mathscr{H}_{|k|}}=\left.\phi\right|_{\mathscr{H}_{k \mid} \mid}, k \geq-1$, that is, $\left.\tilde{\sigma}\right|_{\mathscr{H}}=\phi$. The proof is complete.

To prove the next theorem, we establish the following lemma.

LEMMA 4.5. The natural filtration of $\mathscr{U}$ is invariant under automorphisms of $\mathscr{U}$. 
Proof. Since Der $\mathscr{U}=W$, we have Aut $\mathscr{U}=\operatorname{Aut}(\mathscr{U}: W)$. By [25, Theorem 1], the natural filtration of $W$ is invariant under Aut $W$. Note that $\tilde{\sigma}\left(a D_{i}\right)=\sigma(a) \tilde{\sigma}$, $\sigma \in$ Aut $\mathscr{U}, a \in \mathscr{U}, i \in Y$, which implies the desired result.

Following [20], we introduce some notations. For $X=\mathscr{U}$ or $\mathscr{H}$, put

$$
\begin{aligned}
& \text { Aut }^{*} X=\left\{\sigma \in \text { Aut } X \mid \sigma\left(X_{[j]}\right) \subset X_{[j]}, j \in \mathbb{Z}\right\} ; \\
& \text { Aut }_{i} X=\left\{\sigma \in \text { Aut } X \mid(\sigma-1)\left(X_{j}\right) \subset X_{i+j}, j \in \mathbb{Z}\right\}, \quad i \geq 0 .
\end{aligned}
$$

According to Lemma 4.5 and Corollary 3.5, the natural filtration of $X$ is invariant under Aut $X$. Thus Aut ${ }^{*} X<$ Aut $X$ and Aut ${ }_{i} X \triangleleft$ Aut $X, i \geq 0$. We call Aut $X>$ Aut $_{1} X>$ Aut $_{2} X>\cdots$ the standard normal series of Aut $X$.

Set $\operatorname{Aut}^{*}(\mathscr{U}: \mathscr{H})=\operatorname{Aut}^{*} \mathscr{U} \cap \operatorname{Aut}(\mathscr{U}: \mathscr{H})$ and $\operatorname{Aut}_{i}(\mathscr{U}: \mathscr{H})=\operatorname{Aut}_{i} \mathscr{U} \cap$ Aut $(\mathscr{U}: \mathscr{H})$. We call $\operatorname{Aut}^{*}(\mathscr{U}: \mathscr{H})$ the homogeneous admissible automorphism group of $\mathscr{U}$, and $\operatorname{Aut}_{0}(\mathscr{U}: \mathscr{H})>$ Aut $_{1}(\mathscr{U}: \mathscr{H})>\cdots$ the standard normal series of $\operatorname{Aut}(\mathscr{U}: \mathscr{H})$.

THEOREM 4.6. Suppose that $\Phi$ is defined as in Theorem 4.2. Then

(i) $\Phi\left(\operatorname{Aut}_{i}(\mathscr{U}: \mathscr{H})\right)=\operatorname{Aut}_{i} \mathscr{H}, i \geq 0$;

(ii) $\Phi\left(\right.$ Aut $\left.^{*}(\mathscr{U}: \mathscr{H})\right)=$ Aut $^{*} \mathscr{H}$;

(iii) Aut $\mathscr{H}_{\text {ii }}$ is solvable normal subgroup of Aut $\mathscr{H}$;

(iv) Aut $\mathscr{H}=$ Aut $_{1} \mathscr{H} \rtimes$ Aut $^{*} \mathscr{H}$.

PROOF. (i) We first prove the inclusion ' $C$ '. Let $\sigma \in \operatorname{Aut}_{i}(\mathscr{U}: \mathscr{H})$. Then $\sigma^{-1} \in \operatorname{Aut}_{i}(\mathscr{U}: \mathscr{H})$. For $k \in \mathbb{N}_{0}$ and $f \in \mathscr{U}_{k}$, we may suppose that $\sigma^{-1} f=f+f^{\prime}$, $f^{\prime} \in \mathscr{U}_{i+k}, \sigma\left(D_{j} f\right)=D_{j} f+f^{\prime \prime}, f^{\prime \prime} \in \mathscr{U}_{i+k-1}$. By Lemma 4.5, $\sigma\left(D_{j} f^{\prime}\right) \in \mathscr{U}_{i+k-1}$. Note that

$$
\begin{aligned}
\tilde{\sigma}\left(D_{j}\right)(f) & =\sigma D_{j} \sigma^{-1}(f)=\sigma D_{j}\left(f+f^{\prime}\right) \\
& =\sigma\left(D_{j} f+D_{j} f^{\prime}\right)=D_{j} f+f^{\prime \prime}+\sigma\left(D_{j} f^{\prime}\right) .
\end{aligned}
$$

We obtain that $\tilde{\sigma}\left(D_{j}\right) f \equiv D_{j} f\left(\bmod \mathscr{U}_{i+k-1}\right)$. This implies that $\tilde{\sigma}\left(D_{j}\right) \equiv D_{j}$ $\left(\bmod W_{i-1}\right), j \in Y$. Notice that $\tilde{\sigma}\left(a D_{j}\right)=\sigma(a) \tilde{\sigma}\left(D_{j}\right), j \in Y, a \in \mathscr{U}_{l}$. We may obtain that $\tilde{\sigma}\left(a D_{j}\right) \equiv a D_{j}\left(\bmod W_{i+l-1}\right)$. Therefore $\tilde{\sigma} \in$ Aut $_{i} W$. Thus $\tilde{\sigma} \in$ Aut $_{i} W \cap$ Aut $\mathscr{H} \subset$ Aut $_{i} \mathscr{H}$, and $\Phi\left(\operatorname{Aut}_{i}(\mathscr{U}: W)\right) \subset$ Aut $_{i} W$.

To prove the converse inclusion, suppose that $\varphi \in$ Aut $_{i} \mathscr{H}, i \geq 0$ and set $\sigma:=\Phi^{-1}(\varphi)$. Given $j \in Y$, pick $k \in Y \backslash j^{\prime}$. By Lemma $2.6(\mathrm{i}), \mathrm{T}_{\mathrm{H}}\left(x_{k^{\prime}} x_{j}\right)=$ $(-1)^{\mu\left(k^{\prime}\right)+\mu\left(k^{\prime}\right) \mu(j)} x_{j} D_{k}+(-1)^{\mu(j)} x_{k^{\prime}} D_{j^{\prime}}$. Then

$$
\begin{aligned}
& (-1)^{\mu\left(k^{\prime}\right)+\mu\left(k^{\prime}\right) \mu(j)} \sigma\left(x_{j}\right)\left(\varphi D_{k}\right)+(-1)^{\mu(j)} \sigma\left(x_{k^{\prime}}\right)\left(\varphi D_{j^{\prime}}\right) \\
& \quad=\varphi\left(\mathrm{T}_{\mathrm{H}}\left(x_{k^{\prime}} x_{j}\right)\right) \\
& \quad \equiv(-1)^{\mu\left(k^{\prime}\right)+\mu\left(k^{\prime}\right) \mu(j)} x_{j} D_{k}+(-1)^{\mu(j)} x_{k^{\prime}} D_{j^{\prime}} \quad\left(\bmod \mathscr{H}_{i}\right) .
\end{aligned}
$$


Noticing that $\varphi \in$ Aut $_{i} \mathscr{H}$ and $W_{[-1]}=\mathscr{H}_{[-1]}$, we have

$$
\varphi\left(D_{k}\right)=D_{k}+E_{1}, \quad \varphi\left(D_{j^{\prime}}\right)=D_{j^{\prime}}+E_{2}, \quad \text { where } E_{1}, E_{2} \in \mathscr{H}_{i-1} .
$$

By Lemma 4.5, it is easy to see that $\sigma\left(x_{j}\right) E_{1}, \sigma\left(x_{k^{\prime}}\right) E_{2} \in W_{i}$. Thus we obtain from (13) and (14),

$$
(-1)^{\mu\left(k^{\prime}\right)+\mu\left(k^{\prime}\right) \mu(j)}\left(\sigma\left(x_{j}\right)-x_{j}\right) D_{k}+(-1)^{\mu(j)}\left(\sigma\left(x_{k^{\prime}}\right)-x_{k^{\prime}}\right) D_{j^{\prime}} \equiv 0 \quad\left(\bmod W_{i}\right) .
$$

Since $k \neq j^{\prime}$, we obtain $\sigma\left(x_{j}\right) \equiv x_{j}\left(\bmod \mathscr{U}_{i+1}\right)$. Now using induction on $|\alpha|+|u|$, one may prove that $\sigma\left(x^{(\alpha)} x^{u}\right) \equiv x^{(\alpha)} x^{u}\left(\bmod \mathscr{U}_{|\alpha|+|u|+i}\right)$. This means $\sigma \in$ Aut $_{i} \mathscr{U}$ and therefore $\sigma \in \operatorname{Aut}_{i}(\mathscr{U}: \mathscr{H})$. Hence $\Phi\left(\right.$ Aut $\left._{i}(\mathscr{U}: \mathscr{H})\right) \supset$ Aut $_{i} \mathscr{H}$.

(ii) The proof is completely analogous to (i), therefore is omitted.

(iii) Using the invariance of the natural filtration (see Corollary 3.5), one may verify directly that $\left[\right.$ Aut $_{i} \mathscr{H}$, Aut $\left._{j} \mathscr{H}\right] \subset$ Aut $_{i+j} \mathscr{H}, i, j \geq 0$ (see [19, page 210]). From this we see that the normal series Aut $_{1} \mathscr{H}>$ Aut $_{2} \mathscr{H}>\cdots$ is abelian (that is, Aut $_{i} \mathscr{H} /$ Aut $_{i+1} \mathscr{H}$ are abelian groups, for all $i \geq 1$ ), and reaches 0 . Therefore Aut $_{1} \mathscr{H}$ is solvable.

(iv) Let $\varphi \in$ Aut $\mathscr{H}$. Then there exists $\varphi_{0}, \varphi_{1} \in \operatorname{Hom}_{\mathbb{F}}(\mathscr{H}, \mathscr{H})$ such that $\varphi=\varphi_{0}+$ $\varphi_{1}$ and $\varphi_{0}\left(\mathscr{H}_{[j]}\right) \subset \mathscr{H}_{[j]}, \varphi_{1}\left(\mathscr{H}_{j}\right) \subset \mathscr{H}_{j+1}, j \geq-1$. As the filtration of $\mathscr{H}$ is invariant under Aut $\mathscr{H}$, we have $\varphi_{0} \in$ Aut $^{*} \mathscr{H}$. Therefore, $\varphi_{0}^{-1} \phi=1+\varphi_{0}^{-1} \varphi_{1} \in$ Aut $_{1} \mathscr{H}$. Hence (iv) holds.

Acknowledgement. The authors thank the referee for helpful suggestions.

\section{References}

[1] F. A. Berezin and M. S. Marinov, 'Particle spin dynamics as the grassmann varlant of classical mechanics', Ann. Physics 104 (1977), 336-362.

[2] R. E. Block and R. L. Wilson, 'Classification of the restricted simple Lie algebras', J. Algebra 114 (1988), 115-259.

[3] N. Jacobson, Lie algebras (Interscience, New York, 1962).

[4] N. Jin, 'Ad-nilpotent elements, quasi-nilpotent elements and invariant filtrations of infinitedimensional Lie algebras of Cartan-type', Sci. China Ser. A 35 (1992), 1191-1200.

[5] V. G. Kac, 'On the classification of the simple Lie algebras over a field with nonzero characteristic', Math. USSR Izv. 4 (1970), 391-413.

[6] — 'Description of filtered Lie algebras with which graded Lie algebras of Cartan-type are associated', Math. USSR Izv. 8 (1974), 801-835.

[7] —_ 'Lie superalgebras', Adv. Math. 26 (1977), 8-96.

[8] — , 'Classification of infinite-dimensional simple linearly compact Lie superalgebras', Adv. Math. 139 (1998), 1-55.

[9] Yu. Kochetkov, 'Induced irreducible representations of Leites superalgebras', in: Problem in group theory and homological algebra 139 (Yaroslav. Gos. Univ., Yaroslavl, 1983) pp. 120-123. (in Russian). 
[10] A. I. Kostrikin and I. R. Shafarevic, 'Graded Lie algebras of finite characteristic', Math. USSR Izv. 3 (1969), 237-304.

[11] D. A. Leites, 'New Lie superalgebras and mechanics', Dokl. Akad. Nauk SSSR 236 (1977), 804 807. English translation: Soviet Math. Dokl. (5), 18 (1977), 1277-1280.

[12] __. 'Automorphisms and real forms of simple Lie superalgebras of formal vector fields', in: Problem in group theory and homological algebra 139 (Yaroslav. Gos. Univ., Yaroslavl, 1983) pp. 126-128. (in Russian).

[13] V. M. Petrogradski, 'Identities in the enveloping algebras for modular Lie superalgebras', J. Algebra 145 (1992), 1-21.

[14] A. N. Rudakov, 'Subalgebras and automorphisms of Lie algebras of Cartan-type', Funktsional. Anal. Prilozhen. 20 (1986), 83-84 (in Russian).

[15] M. Scheunert, Theory of Lie superalgebras, Lectures Notes in Math. 716 (Springer, Berlin, 1979).

[16] G.-Y. Shen, 'An intrinsic property of the Lie algebra $K(m, n)$ ', Chin. Ann. Math. 2(1981), 104-107.

[17] H. Strade, 'The classification of the simple modular Lie algebras: IV. Determining of the associated graded algebra', Ann. of Math. (2) 138 (1993), 1-59.

[18] H. Strade and R. Farnsteiner, Modular Lie algebras and their representations (Marcel Dekker, 1988).

[19] R. L. Wilson, 'Classification of generalized Witt algebras over algebraically closed fields', Trans. Amer. Math. Soc. 153 (1971), 191-210.

[20] — 'Automorphisms of graded Lie algebras of Cartan type', Commun. Algebra 3 (1975), $591-613$.

[21] — 'A structural characterization of the simple Lie algebras of generalized Cartan-type over fields of prime characteristic', J. Algebra 40 (1976), 418-465.

[22] __, 'Simple Lie algebras of type S', J. Algebra 62 (1980), 292-298.

[23] Y.-Z. Zhang, 'Finite-dimensional Lie superalgebras of Cartan-type over fields of prime characteristic', Chin. Sci. Bull. 42 (1997), 720-724.

[24] Y.-Z. Zhang and H.-C. Fu, 'Finite-dimensional hamiltonian Lie superalgebras', Commun. Algebra 30 (2002), 2651-2673.

[25] Y.-Z. Zhang and J.-Z. Nan, 'Finite-dimensional Lie superalgebras $W(m, n, t)$ and $S(m, n, t)$ of Cartan-type', Chin. Adv. Math. 27 (1998), 240-246.

[26] Y.-Z. Zhang and G.-Y. Shen, 'The embedding theorem of $\mathbb{Z}$-graded Lie superalgebras', Sci. China Ser. A 41 (1998), 1009-1016.

Department of Mathematics

Harbin Normal University

Harbin 150080

China

and

Department of Mathematics

Northeast Normal University

Changchun 130024

China

e-mail: wendeliu@sohu.com
Department of Mathematics Harbin Normal University

Harbin 150080

China

e-mail: zhyz@nenu.edu.cn 